\title{
ESTRATEGIA DIDÁCTICA PARA CONTRIBUIR A LA EDUCACIÓN PARA EL DESARROLLO SOSTENIBLE EN LA ENSEÑANZA PREUNIVERSITARIA
}

\author{
Alicia Lauzardo'; Varinia Paredes²; J .M. Aparicio ${ }^{3}$ \\ ${ }^{1}$ Docente investigadora. Instituto Superior Pedagógico “Rubén Martínez Villena”. La Habana. Cuba. E-mail: yaniracu@yahoo.es \\ ${ }^{2}$ Profesora Titular. Departamento Medicina Veterinaria. Facultad de Ciencia Animal. Universidad Nacional Agraria de Nicaragua. FACA/ \\ UNA. E-mail: varinia.paredes@una.edu.ni \\ ${ }^{3}$ Profesor Titular. Facultad de Medicina Veterinaria. Universidad Agraria de la Habana, Cuba. FMV / UNAH. km 1, San José de las Lajas, \\ provincia La Habana, E-mail yaimelcu@yahoo.es; aparicio@isch.edu.cu.
}

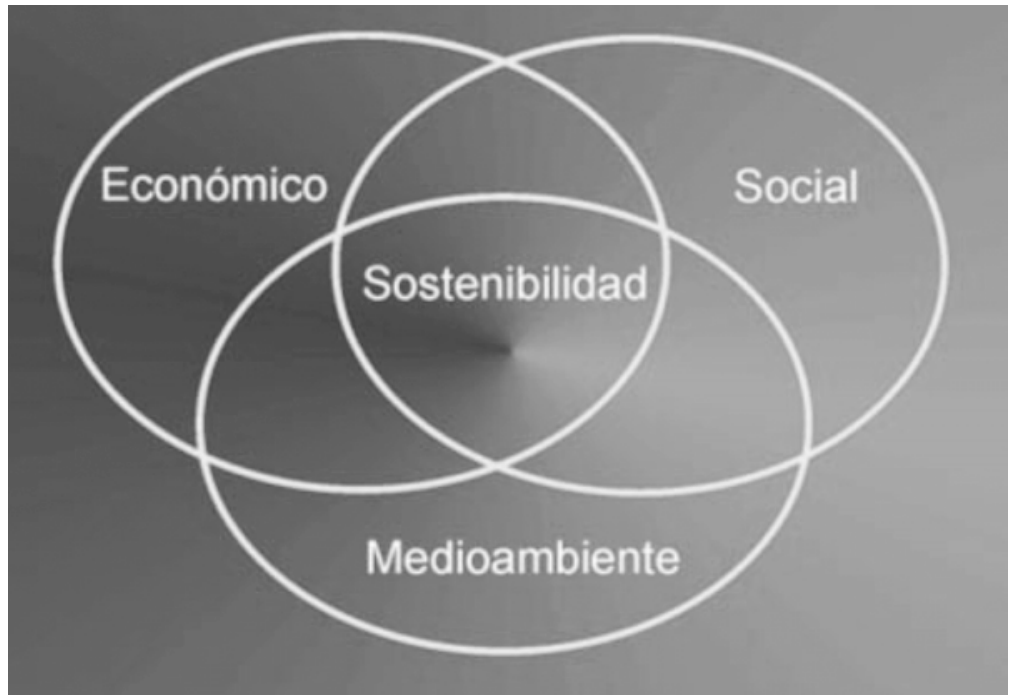

\section{RESUMEN}

Dentro de los desafíos a los que debe hacer frente la humanidad para hacer posible un futuro sostenible, se encuentra, entre otros, el de impulsar una educación solidaria, que contribuya a una correcta percepción del mundo y prepare a los ciudadanos para la toma de decisiones fundamentadas e impulse su comportamiento a lograr un desarrollo culturalmente plural y físicamente sostenible. Los educadores están llamados a formar ciudadanos y ciudadanas conscientes de la gravedad y el carácter global de los problemas y prepararlos para la toma de decisiones adecuadas. La estrategia didáctica para contribuir a la Educación para el desarrollo sostenible desde las asignaturas de la enseñanza preuniversitaria en Cuba pretende ir sentando las bases para que esto sea posible y lograr modestamente un aprendizaje reflexivo, crítico y desarrollador en el cual el estudiante se convierta en el protagonista de su propio aprendizaje. La estrategia puesta en práctica durante dos cursos arrojó como resultados la sensibilización creciente sobre el problema de la sustentabilidad y su vinculación con los problemas sociales, económicos, culturales y tecnológicos. Consolidó competencias para el trabajo en equipos y la elaboración de informes y se aprecia que los estudiantes emiten juicios críticos y autocríticos ante los problemas para la sostenibilidad.

Palabras clave: Educación, didáctica, sostenibilidad, química, medio ambiente

\begin{abstract}
Among the challenges that mankind must face to build a possible sustainable future is to impulse a friendship education that contribute to reach a right perception of the world to allow taking justified decisions and impulse to achieve, behavior and a wide cultural development. Educators are demanded to build conscious citizens to face the actual serious globalized situation of the world. The didactic strategy to contribute from education in the subject of chemistry in Cuban student high school, tries to create the basis that permit a critical reflexive learning that conceives the student to become the leadership of the process. The trial carried out during the last two courses had good results because it was observed a change in the point of view in the way things were managed, but also in connection with social, economical, cultural and technological problems. It was concluded that students were able to deliver workshops, make their own critical judgments and to afford new challenges facing problems in connection with sustainable development.
\end{abstract}




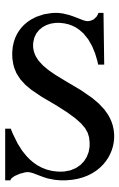
e vive una situación de auténtica emergencia planetaria, expresión acuñada por el biólogo Richard Bybee (1991) para llamar la atención sobre el conjunto de problemas de alcances globales y estrechamente relacionados a los que la humanidad ha de hacer frente (Vilches y Gil, 2003).

En el compromiso "Por una educación para la sostenibilidad" de las Naciones Unidas se plantea que esta situación de auténtica emergencia planetaria es el resultado de comportamientos individuales y colectivos orientados a la búsqueda de beneficios particulares y a corto plazo, sin atender sus consecuencias para los demás o para las futuras generaciones. Una actitud criticable por razones éticas y por constituir la expresión de un egoísmo poco inteligente, que no toma en consideración las consecuencias, para nosotros mismos, de las acciones guiadas por intereses particulares inmediatos.

La gravedad de esta situación ha dado lugar a numerosos llamamientos a la ciudadanía en general y a las comunidades tecno científicas y educativas en particular, dado el efecto multiplicador que su implicación puede tener en la formación de ciudadanas y ciudadanos conscientes de la situación y preparados para participar en la necesaria toma de decisiones para hacerle frente.

Se puede mencionar el llamamiento realizado a fines del Siglo XX por Jane Lubchenco, bióloga y presidenta de la American Association for the Advancement of Science y editora de la prestigiosa revista Science. Lubchenco reclamaba que el siglo XXI fuera, para la ciencia, el siglo del medio ambiente y que la comunidad científica reorientara su maquinaria hacia la resolución de los problemas que amenazan el futuro de la humanidad.

Por lo que respecta a los educadores, los llamamientos se vienen prodigando desde que en 1972 las Naciones Unidas organizaron en Estocolmo la Conferencia del Medio Ambiente Humano.

Particular repercusión en los medios tuvo la Primera Cumbre de la Tierra, celebrada en Río de Janeiro en 1992, en la que se pidió a los educadores de todas las áreas y niveles, tanto de la educación formal como de la no reglada, que contribuyeran a una formación ciudadana para hacer posible un futuro sostenible.

Se reconoce por parte de algunos autores que tanto la mayoría de los científicos como los educadores no respondían, en general, a estos llamamientos y por ello, 10 años después, en la Segunda Cumbre de la Tierra celebrada en Johannesburgo, se comprendió la necesidad de una campaña intensa y de larga duración. Surgió así la idea de una Década de la Educación por un Futuro Sostenible para el período 2005-2014, destinada a lograr la implicación de todos los educadores en la formación de una ciudadanía atenta a la situación del planeta y prepa- rada para la necesaria toma de decisiones (Resolución 57/254 aprobada por unanimidad en la Asamblea General de las Naciones Unidas el 20 de Diciembre de 2002).

\section{DESARROLLO}

¿Cuál es el papel que le corresponde desempeñar a la educación?

La UNESCO plantea que el papel de la educación para un desarrollo humano sostenible es el de contribuir a hacer que todo esto sea posible. La educación y la formación aparecen como factores determinantes para el incremento de la creatividad y la racionalidad, la habilidad para resolver problemas y la competencia necesaria para fomentar las cada vez más complejas decisiones de tipo cultural, social y tecnológico que conlleva un desarrollo sostenible. Reformar el sistema educativo para cumplir estos objetivos significa que los decidores tendrán que enfrentarse al menos a dos retos importantes: la creación de estrategias educativas institucionales y programas que tengan en cuenta todos los posibles actores educativos y el incremento de la calidad y utilidad de los diferentes procesos educativos y de formación dirigidos principalmente a los ciudadanos y a los jóvenes.

Se deben crear los medios y la forma más efectiva de capacitar a sus ciudadanos para que contribuyan al objetivo común de mejorar las condiciones de vida material y espiritual de las generaciones actuales, sin privar a generaciones venideras de una vida digna.

La función de una educación para un desarrollo sostenible es, fundamentalmente, la de desarrollar el capital humano y potenciar el progreso tecnológico, así como crear las condiciones culturales que propicien el cambio social y económico.

Existen hoy dos retos importantes a la hora de reformar la educación para que esta contribuya a un desarrollo sostenible: Desarrollar nuevas formas de organizar el proceso educativo, utilizando todos los recursos educativos potenciales de la sociedad, y el desarrollo de nuevos programas, metodologías y enfoques que ayuden a los ciudadanos que deben tomar y poner en práctica decisiones a todos los niveles y resolver de manera efectiva los problemas cada vez más complejos que plantea un desarrollo humano sostenible .

Según la propia fuente una remodelación de la educación que favorezca el desarrollo sostenible, que suscite el desarrollo de una concienciación y de un compromiso moral, requiere de una acción coordinada que abarca una amplia gama de actores, canales de comunicación y entornos de aprendizaje. Sin embargo, la pluralidad de actores y de mecanismos no puede reemplazar el papel de la educación. Solo un proceso de aprendizaje organizado y orientado hacia objetivos puede transformar la infor- 
mación en conocimiento y convertirlos en herramientas útiles para el progreso técnico y el cambio social.

Se plantea en los materiales consultados que el contenido de la educación para un desarrollo sostenible puede obtenerse de una forma más eficaz a través de las nuevas tecnologías de información y comunicaciones. Las crecientes cantidades de información disponible, la corta vida del conocimiento y la cada vez mayor complejidad de los fenómenos requiere equipar a los individuos con herramientas intelectuales de alcance general, que los faculten para explorar de manera permanente e interdisciplinaria los problemas nacionales y globales.

La situación actual que muestra el mundo y en especial América Latina, resalta la imperiosa necesidad de elevar la cultura ambiental y para el desarrollo sostenible de las nuevas generaciones y es incuestionable que esa responsabilidad recae básicamente en la escuela, desde donde, de conjunto con la familia y las diferentes entidades de la comunidad, se prepara a los ciudadanos de hoy y del futuro.

Existe una política sobre el medio ambiente bien definida en algunos países, incluso dentro de la Constitución de algunos y en correspondencia, en los documentos que rigen las actividades que se organizan en instituciones de algunos Estados.

Algunos documentos normativos plantean que el objetivo de la educación es la formación integral de las nuevas generaciones, por lo que el papel de los educadores es propiciar la preparación de los estudiantes en todas las esferas del saber, en correspondencia con los intereses de la sociedad actual.

La educación tiene el propósito de formar un hombre que participe activamente en la edificación de la sociedad contemporánea, con un alto nivel de responsabilidad, habilidades prácticas, sentimientos humanos y valores estéticos y ético-morales, lo que implica en definitiva, formar un hombre revolucionario y culto.

La escuela, institución encargada de la formación integral de los ciudadanos debe garantizar que los estudiantes se apropien de los conocimientos, las habilidades y los valores que les sirvan de base teórica para orientar su conducta y comportamiento en bienestar del cuidado y la protección del medio ambiente en que se desarrolla, posibilitando, además, que se promueva una participación activa a favor de un desarrollo sostenible.

¿Qué sucede en la enseñanza preuniversitaria en relación con la educación para el desarrollo sostenible?

En las actuales transformaciones en la Enseñanza Preuniversitaria existen prioridades que se relacionan a continuación: Lograr una formación integral y armónica de los estudiantes, contribuir a la formación de estilos de vida saludables, aprender a trabajar con las modernas TIC, independencia en el trabajo con los textos para que puedan dominar los adelantos de la ciencia y la técnica. En el trabajo con los profesores conocer donde están las dificultades de los estudiantes y ayudarlos a resolver los problemas durante el proceso docente educativo para así elevar su aprendizaje.

Partiendo de estas prioridades y teniendo en cuenta la necesidad de formar ciudadanos activos, competentes, creativos y racionales, con habilidades para resolver problemas y la competencia necesaria para fomentar las cada vez más complejas decisiones, sin lugar a dudas hay que plantearse este problema científico

¿Cómo contribuir a la educación para el desarrollo sostenible a partir de las asignaturas que cursan los estudiantes en la educación preuniversitaria?

En principio, corresponde aclarar que hoy en día el concepto o noción del Desarrollo Sostenible no es fijo e invariable, ya que el Desarrollo Sostenible puede ser entendido como un proceso conducente a una profunda reflexión, revisión y cambio sustancial en las interrelaciones e interacciones entre los sistemas y procesos sociales, económicos y naturales. El estado actual de esas relaciones nos plantea, para nuestra supervivencia, el desafío de armonizar y equilibrar las demandas del progreso económico y social con la salvaguarda y conservación de los ecosistemas mundiales que sustentan la vida. Dadas las numerosas definiciones y descripciones del Desarrollo Sostenible, algunas muy complejas, para contribuir a consolidar su conceptualización, se repasan seguidamente distintas nociones alusivas:

En el informe Nuestro futuro común, de la Comisión Mundial para el Ambiente y el Desarrollo, ONU, de 1987, se define al desarrollo sostenible como una forma de progreso que satisface las necesidades del presente sin comprometer la capacidad de las generaciones venideras de satisfacer sus propias necesidades.

La Ley 81/1997 del Medio Ambiente en Cuba expresa que Desarrollo Sostenible es un proceso de elevación sostenida y equitativa de la calidad de vida de las personas, mediante el cual se procura el crecimiento económico y el mejoramiento social, en una combinación armónica con la protección del medio ambiente, de modo que se satisfacen las necesidades de las actuales generaciones, sin poner en riesgo la de futuras generaciones. Para el desarrollo del presente trabajo se asumió que para contribuir al desarrollo sostenible desde la educación, significa asumir una posición más crítica, analítica y participativa, donde el sujeto tenga una posición activa frente al conocimiento y sea capaz de generar cambios 
en la vida actual sin comprometer las condiciones de las generaciones futuras.

Constituye una cualidad del hombre los sentimientos de cuidado, amor y conservación del medio ambiente, sin embargo, esto no se desarrolla espontáneamente, es necesaria la influencia, la orientación, la educación de la sociedad en general, de la comunidad en lo particular y de la familia en el plano de lo singular. Lugar especial tiene la escuela en este sistema de influencias, pues es la institución especializada con que cuenta la sociedad para formar estos valores que resultan imprescindibles en una personalidad multilateral y armónicamente desarrollada.

Es incuestionable que "El desarrollo sustentable requiere un enfoque holístico: la educación para el desarrollo sustentable tiene vínculos con otros programas en la educación. No es un nuevo programa sino un proceso de reorientación para que la educación desempeñe su papel en construir las capacidades de todos los miembros de la sociedad para trabajar conjuntamente y edificar un futuro sostenible". (UNESCO, 2004).

Particularmente en relación con el área de la Educación Ambiental y Educación para un Desarrollo Sostenible, se reafirma la necesidad, ahora más que nunca, de que los educadores, con el debido y conveniente apoyo, efectúen, conforme a sus posibilidades, una constante actualización, revisión y enriquecimiento del enfoque, los objetivos, los contenidos y otros aspectos, curriculares y extracurriculares, relacionados con los procesos de enseñanza-aprendizaje que tienen habitualmente a su cargo.
¿Qué se propone?

Una propuesta para el campo del desarrollo sostenible, puede apoyarse en muchos elementos de la investigación participativa, la que, como variante de investigación - acción, hace confluir tres procesos: el investigativo, el educativo y el de desarrollo y transformación de la realidad.

Por todo lo anteriormente expuesto se propone con este trabajo una estrategia didáctica que contribuya por la vía curricular y extracurricular al desarrollo de jóvenes capaces de asumir los retos que impone el desarrollo sostenible.

La propuesta incluye seminarios integradores, proyectos de investigación, estudio integral de la localidad, noticieros ambientalistas, reflexiones y debates de videos y artículos de la prensa escrita, concursos de fotografías, tareas integradoras, todos ellos vinculados con los contenidos de las asignatura y con una visión interdisciplinaria en el área de las ciencias naturales.

Los seminarios integradores se orientan al comienzo de cada unidad para que el estudiante disponga de tiempo para su autopreparación y pueda visitar la biblioteca, consultar los materiales en soporte digital e indagar en otras fuentes actualizadas sobre los aspectos a investigar; al final de la unidad, en una de las clases de consolidación se organiza el debate en forma de paneles en el aula donde se exponen los resultados de la investigación y se hacen las correcciones si fueran necesarias. En la tabla 1, se presenta una propuesta de seminarios integradore

Tabla 1. Seminarios integradores que contribuyen a la educación para el desarrollo sostenible

\begin{tabular}{|l|l|l|}
\hline Unidad 1: Las disoluciones & $\begin{array}{l}\text { Título: El agua como recurso } \\
\text { natural. Las disoluciones. La } \\
\text { importancia del agua para la } \\
\text { vida. }\end{array}$ & $\begin{array}{l}\text { Objetivo: Valorar la situación actual del recurso natural } \\
\text { agua y su importancia para la vida, con la finalidad de } \\
\text { tomar conciencia sobre la necesidad de su protección } \\
\text { para contribuir a atenuar los efectos nocivos que el propio } \\
\text { hombre ejerce sobre ella. }\end{array}$ \\
\hline Unidad 2: Equilibrio molecular & $\begin{array}{l}\text { Título: El equilibrio molecu- } \\
\text { lar en la naturaleza. } \mathrm{O}_{2(\mathrm{~g})} \mathrm{O}_{3(\mathrm{~g})}\end{array}$ & $\begin{array}{l}\text { Objetivo: Valorar la importancia del equilibrio di oxígeno } \\
\text { - ozono en la atmósfera para la vida en el planeta. }\end{array}$ \\
\hline Unidad 3: Equilibrio iónico & $\begin{array}{l}\text { Título: El pH Importancia del } \\
\text { control del pH de los suelos, el } \\
\text { agua y los líquidos corporales. }\end{array}$ & $\begin{array}{l}\text { Objetivos: Valorar la importancia del control del pH en la } \\
\text { mayoría de los procesos naturales, así como los perjuicios } \\
\text { que ocasionan las lluvias ácidas. }\end{array}$ \\
\hline
\end{tabular}


Los proyectos de investigación que se proponen (Tabla 2), salen por la vía extracurricular a través de las sociedades científicas, estos se agrupan en sociedades que tienen de 5 ó 6 estudiantes y desde el comienzo del curso escogen los temas sobre los que van a investigar, para el evento de sociedades científicas del centro llevan el informe escrito de su investigación y exponen los resultados al resto de sus compañeros.

Los noticieros ambientalistas son responsabilidad de un grupo de estudiantes que divulgarán entre sus compañeros, a través de la radio o de los matutinos, los problemas medio ambientales y de sostenibilidad que se ofrecen en los medios de difusión masiva o en alguna consulta específica que realicen por otros medios como parte de la investigación en los seminarios o en los proyectos de investigación.

Las reflexiones y debates se van proponiendo durante todo el desarrollo del curso por la vía extracurricular, haciendo coincidir estas con fechas significativas como el Día Mundial del Medio Ambiente, el Día Mundial de la Biodiversidad, el Día Internacional de la Paz, etc. Las tareas integradoras y software se proponen a medida que se van desarrollando el currículo de las asignaturas durante todo el curso.

Para el desarrollo de estos seminarios y proyectos de investigación además de las fuentes bibliográficas consultadas, se realizan visitas a centros de investigaciones, entrevistas a investigadores y personal especializado. Se les solicita a los estudiantes que en todos casos se elaboren fichas de contenidos para contribuir a desarrollar habilidades investigativas, puede considerarse que con esta propuesta se contribuye a lograr un aprendizaje reflexivo, crítico y desarrollador en el cual el estudiante es el protagonista de su propio aprendizaje y el profesor un mediador fundamental en el mismo.

Con la discusión en grupos se tratan de modificar las actitudes personales y colectivas y sirve para preparar a las personas para el trabajo en equipo, además de que contribuye al ejercicio de la opinión y la responsabilidad individual. Al proponerles los seminarios integradores y los proyectos de investigación, se preparan para el manejo de técnicas sencillas o complejas de indagación e investigación y consolida las competencias para el trabajo en equipo y la elaboración de informes.

Tabla 2. Proyectos de investigación propuestos para la educación en desarrollo sostenible

\begin{tabular}{|c|c|}
\hline $\begin{array}{l}\text { Tema 1.- El medio ambiente y } \\
\text { sus problemas. }\end{array}$ & $\begin{array}{l}\text { - Globalización y medio ambiente. El desarrollo sostenible; posibilidades. } \\
\text { Pobreza y desarrollo desigual. }\end{array}$ \\
\hline $\begin{array}{l}\text { Tema 2.- El cambio climático } \\
\text { global. }\end{array}$ & $\begin{array}{l}\text { - El efecto invernadero, causas y consecuencias. La actualidad del efecto } \\
\text { invernadero. Cuba y las consecuencias del cambio climático. Responsabilidad } \\
\text { de los países. }\end{array}$ \\
\hline $\begin{array}{l}\text { Tema 3.- El agotamiento del } \\
\text { ozono estratosférico. }\end{array}$ & $\begin{array}{l}\text { - Formación del ozono en la naturaleza. Causas y consecuencias del deterioro de } \\
\text { la capa de ozono. El Protocolo de Montreal. La estrategia cubana. }\end{array}$ \\
\hline $\begin{array}{l}\text { Tema 4.- La diversidad } \\
\text { biológica. }\end{array}$ & $\begin{array}{l}\text { - La pérdida de la diversidad biológica. La biodiversidad como base de la } \\
\text { existencia humana. La disminución y desaparición de especies. Causas. Los } \\
\text { bosques. Los animales amenazados. La sobreexplotación de los recursos } \\
\text { marinos. El problema de la agricultura. La producción de alimentos. Los } \\
\text { biocombustibles. La riqueza natural cubana, su estado actual, perspectivas. La } \\
\text { biodiversidad humana, la paz, la tolerancia. }\end{array}$ \\
\hline $\begin{array}{l}\text { Tema 5.- La desertificación y } \\
\text { los procesos asociados. }\end{array}$ & $\begin{array}{l}\text { - deforestación, erosión de los suelos, escasez del agua en el planeta. La } \\
\text { deforestación, causas y consecuencias. La deforestación en los países } \\
\text { subdesarrollados. La pobreza, las desigualdades sociales y el hambre. La erosión } \\
\text { del suelo, causas, medidas para evitarla. La salinización de los suelos. Situación } \\
\text { en nuestro país. Problemas que produce la salinización de los suelos. El } \\
\text { comportamiento global con relación a la deforestación, la erosión de los suelos y } \\
\text { las desigualdades sociales. }\end{array}$ \\
\hline $\begin{array}{l}\text { Tema 6.- La contaminación del } \\
\text { aire, el agua y las tierras. }\end{array}$ & $\begin{array}{l}\text { - La contaminación del aire: natural y andrógena, quema de combustibles fósiles. } \\
\text { El organismo humano y la contaminación del aire. Las lluvias ácidas. Las lluvias } \\
\text { ácidas. Contaminación de las aguas, desechos peligrosos. El agua el manejo del } \\
\text { agua, el agua de uso doméstico, su ahorro. Los recursos hídricos en tú país y en } \\
\text { tú localidad. Las guerras y la contaminación. Efectos negativos de las guerras. }\end{array}$ \\
\hline $\begin{array}{l}\text { Tema 7.- Las fuentes } \\
\text { renovables de energía. }\end{array}$ & $\begin{array}{l}\text { - La política global en relación con la búsqueda de fuentes renovables de energía. } \\
\text { La reducción del consumo de energía. Las medidas que se pueden adoptar en los } \\
\text { hogares con este fin. }\end{array}$ \\
\hline
\end{tabular}


Dentro de las actividades que se proponen ya fueron puestas en práctica en cursos anteriores los seminarios integradores y proyectos de investigación, los cuales trajeron alentadores resultados en los estudiantes que se vincularon directamente a investigar sobre temas medulares a nivel global y también la incidencia a nivel regional, y hasta en su localidad. En la Tabla 3, se proponen algunos estudios integrales sobre la localidad.

El grado de sensibilidad de los estudiantes frente a los problemas del desarrollo sostenible se valoraron teniendo en cuenta los siguientes indicadores: El juicio de valor que formula el estudiante sobre el problema que percibe. el grado de conocimiento que tiene de la problemática, El interés personal en el tema, la medida en la cual el problema y su resolución son sentidos, el sentido del deber, la noción de justicia y equidad que posee (adaptados de los referentes de Tietelbaun, 1978).

Tabla 3. Estudios integrales en la localidad

- Situación geográfica del territorio. Extensión y límites.

- Población. Características.

- Economía. Principales sectores.

- Educación.

- Cultura.

- Principales logros en el sector de la salud.

- Principales industrias. Elementos de su influencia

en el medio ambiente de su territorio.

- Acueducto y alcantarillado. Vertimiento de desechos.

- Flora y fauna del lugar.
Todos estos indicadores fueron valorados utilizando la técnica del diferencial semántico en la que el estudiante marcaba en una escala de adjetivos bipolares su significado evaluativo de los aspectos que se le plantearon en encuestas y entrevistas.

\section{CONCLUSIONES}

¿Cuáles son los resultados? Los resultados de esta estrategia se van observando a largo plazo, en estos momentos se aprecian algunos:

Los estudiantes muestran interés por conocer acerca del medio ambiente y los problemas asociados a él y son capaces de aportar juicios de valor sobre estas problemáticas a nivel global, regional y local.

Se muestran, en muchos casos, críticos y autocríticos sobre sus conductas y posiciones frente a los retos que se enfrenta hoy la sociedad y se plantean algunas iniciativas para resolver problemas ambientales de su localidad.

Se observa, en el debate que se promueve con las actividades, que los estudiantes adoptan posiciones de justicia y equidad ante la pobreza, la marginalidad, el hambre, las enfermedades, las guerras y otras desigualdades a las que están sometidas muchas personas en el mundo.

Consolida competencias para el trabajo en equipos y la elaboración de informes así como para identificar y formular problemas a través del aprendizaje interdisciplinario.

Como parte de la propuesta además se ofrecen orientaciones metodológicas para organizar y desarrollar las actividades que se proponen como una forma de ayuda a los profesores que van a ponerla en práctica.

\section{REFERENCIAS BIBLIOGRÁFICAS}

CITMA. 1997. Estrategia Nacional de Educación Ambiental. Centro de Información, Gestión y Educación ambiental. La Habana, Cuba;

CITA. 1997. Ley 81 sobre el Medio Ambiente. Cuba; .

Gil Pérez, D; Vilches, A; Toscano, JC. Macias, O. 2006. Década de la Educación para un Futuro Sostenible (20052014): un punto de inflexión necesario en la atención a la situación del planeta. Revista Iberoamericana de Educación 40. P.125-178.

MINED. 2006. Programas de Educación Preuniversitaria. Onceno grado. Ed. Pueblo y Educación, Cuba; p.120131.

UNESCO. 1992. Remodelar la Educación hacia un desarrollo sostenible. Medio Ambiente y desarrollo: Notas, p, 1-16.

Vilches, A; Gil, D. 2008. Obstáculos que dificultan la implicación de los educadores a la educación para la sostenibilidad. V Congreso Internacional de Didáctica de la ciencia. La Habana. 